doi:10.13108/2019-11-4-108

\title{
ON TRIPLE DERIVATIONS OF PARTIALLY ORDERED SETS
}

\author{
A.Y. ABDELWANIS
}

\begin{abstract}
In this paper, as a generalization of derivation on a partially ordered set, the notion of a triple derivation is presented and studied on a partially ordered set. We study some fundamental properties of the triple derivation on partially ordered sets. Moreover, some examples of triple derivations on a partially ordered set are given. Furthermore, it is shown that the image of an ideal under triple derivation is an ideal under some conditions. Also, the set of fixed points under triple derivation is an ideal under certain conditions. We establish a series of further results of the following nature. Let $(P, \leqslant)$ be a partially ordered set.

1. If $d, s$ are triple derivations on $P$, then $d=s$ if and only if $\operatorname{Fix}_{d}(P)=\operatorname{Fix}_{s}(P)$.

2. If $d$ is a triple derivation on $P$, then, for all $x \in P ; \operatorname{Fix}_{d}(P) \cap l(x)=l(d(x))$.

3 . If $d$ and $s$ are two triple derivations on $P$, then $d$ and $s$ commute.

4. If $d$ and $s$ are two triple derivations on $P$, then $d \leqslant s$ if and only if $s d=d$.

In the end, the properties of ideals and operations related to triple derivations are examined.
\end{abstract}

Keywords: Triple derivation; fixed point; ideal; partially ordered set.

Mathematics Subject Classification: 06E20; 13N15

\section{INTRODUCTION}

The concept of derivations has appeared in various researches and was studied by many mathematicians. It also circulated in many mathematical concepts such as Jordan triple derivations, generalized Jordan triple derivations, Jordan triple higher derivations, generalized Jordan triple higher derivations, generalized Lie triple higher derivations and multiplicative $*$-lie triple higher derivations. There is a lot of scientific researchs devoted to the generalizations of the concept of derivations. For example, the concepts of Jordan triple derivations and generalized Jordan triple derivations appeared in [6], [7, [9]. The concepts of Jordan triple higher derivations and generalized Jordan triple higher derivations appeared also in [8]; for the other concepts see [1], 2].

The concept of derivations on a lattice appeared in [3] and [11]. Later, in 2014, the notion of derivations was generalized to the notion of triple derivations on lattices as in the following definition.

Definition 1.1. Let $(L, \wedge, \vee)$ be a lattice and $d: L \rightarrow L$ be a function. We call $d$ a triple derivation on $L$ if it satisfies the following condition:

$d(x \wedge y \wedge z)=(d(x) \wedge y \wedge z) \vee(x \wedge d(y) \wedge z) \vee(x \wedge y \wedge d(z))$ for all $x, y, z \in L$.

In 2017, the notion of derivation of partially ordered sets was presented in [12] as follows.

Definition 1.2. Let $P$ be a poset and $d: P \rightarrow P$ be a function. We call $d$ a derivation on $P$ if it satisfies the following conditions:

(1) $d(l(x, y))=l(u(l(d(x), y), l(x, d(y))))$ for all $x, y \in P$;

(2) $l(d(u(x, y)))=l(u(d(x), d(y)))$ for all $x, y \in P$.

A.Y. Abdelwanis, On triple Derivations of Partially ordered SETS.

(C) Abdelwanis A.Y. 2018.

Submitted November 15, 2018. 
In this present paper, the notion of triple derivation of a partially ordered set is introduced and some related properties are studied for the triple derivation on a partially ordered set.

We introduce a few notations, which will be employed in what follows. A pair $(P, \leqslant)$ always denotes a partially ordered set (poset). We additionally employ the shorthand notation $P$ to indicate a poset. For $y \in P$, as in [12], we define $\downarrow y=\{p \in P: p \leqslant y\}$ and $\uparrow y=\{p \in P: y \leqslant$ $p$. For $B \subseteq P$, we denote by

$$
l(B)=\{p \in P: p \leqslant b \text { for all } b \in B\}
$$

the lower cone of $B$ and

$$
u(B)=\{p \in P: b \leqslant p \text { for all } b \in B\}
$$

is the upper cone. We immediately see that both are antitone and their compositions $l(u(\cdot))$ and $u(l(\cdot))$ are monotone. Also we have $l(u(l(\cdot)))=l(\cdot), u(l(u(\cdot)))=u(\cdot)$ from [4]. If $B=$ $\left\{b_{1}, b_{2}, \ldots, b_{n}\right\}$ is a finite subset, then we simply write $l(B)=l\left(b_{1}, b_{2}, \ldots, b_{n}\right)$ and $u(B)=$ $u\left(b_{1}, b_{2}, \ldots, b_{n}\right)$. Also, for $A \subseteq P$ and $B \subseteq P$, we will denote $l(A, B)$ for $l(A \cup B)$ and $u(A, B)$ for $u(A \cup B)$. For $A \subseteq P$, we write $\downarrow A=\{p \in P: p \leqslant a$ for some $a \in A\}$. According [5], if $A=\downarrow A$, then $A$ is said to be a lower set. $A$ is directed if it is nonempty and every finite subset of $A$ has an upper bound in $A$; thanks to the nonemptiness, it is reasonable to expect that each combination of components in $A$ has an upper bound in $A$. A subset $J$ of $P$ is called an ideal if it is a directed lower set.

In this paper, in Section 2, we present the notion of triple derivations of partially ordered sets and discuss their essential properties. In Section 3, we examine the fixed sets from the point of view of the triple derivation. In Section 4, we examine the properties of ideals and the operations related to the triple derivation. Our results generalize the results of [12].

\section{TRIPLE DERIVATIONS OF POSETS}

Definition 2.1. Let $P$ be a poset and $d: P \rightarrow P$ be a map. We call $d$ a triple derivation on $P$, if it satisfies the following conditions:

$1 d(l(x, y, z))=l(u(l(d(x), y, z), l(x, d(y), z), l(x, y, d(z)))$ for all $x, y, z \in P$;

$2 l(d(u(x, y, z)))=l(u(d(x), d(y), d(z)))$ for all $x, y, z \in P$.

Remark 2.1. Suppose $P=(P, \leqslant, \wedge, \vee)$ is a lattice, then we can prove that if $d$ is a triple derivation on $(P, \leqslant)$, then $d$ is a triple derivation on lattice $(P, \wedge, \vee)$.

Lemma 2.1. Let $P$ be any poset, then any surjective derivation on $P$ is a triple derivation on $P$.

Proof. Let $(P, \leqslant)$ be a poset and $d: P \rightarrow P$ be a surjective derivation. First we are going to show that

$$
d(l(x, y, z))=l(u(l(d(x), y, z), l(x, d(y), z), l(x, y, d(z))) \quad \text { for all } \quad x, y, z \in P .
$$

First we prove that

$$
d(l(x, y, z)) \subseteq l(d(x), y, z) \quad \text { for all } \quad x, y, z \in P .
$$

Let $x, y, z \in P$ and $s \in d(l(x, y, z))$, then there exist $t \in l(x, y, z)$ such that $s=d(t)$. Since $d$ is a derivation on $P$, then by Statement 1 in Proposition 2.1 in [12] we get $s=d(t) \leqslant t$. But $t \in l(x, y, z)$ and $d$ is a derivation then again by using Statements 1, 2 in Proposition 2.1 of [12], we obtain

Hence, we have

$$
s \leqslant d(x), \quad s \leqslant d(y) \leqslant y, \quad s \leqslant d(z) \leqslant z .
$$

$$
s \leqslant d(x), \quad s \leqslant y, \quad s \leqslant z,
$$


i.e. $s \in l(d(x), y, z)$. Therefore,

$$
d(l(x, y, z)) \subseteq l(d(x), y, z)=l(u(l(d(x), y, z))) \subseteq l(u(l(d(x), y, z), l(x, d(y), z), l(x, y, d(z))) .
$$

On the other hand, since $d$ is a surjective derivation on $P$, we see easily that

$$
l(d(x), y, z)=l(x, d(y), z)=l(x, y, d(z)) \quad \text { for all } \quad x, y, z \in P .
$$

Then

$$
l(u(l(d(x), y, z), l(x, d(y), z), l(x, y, d(z)))=l(u(l(d(x), y, z)))=l(d(x), y, z)
$$

for all $x, y, z \in P$. But $d$ is a derivation and we find $l(d(x), y, z) \subseteq l(x, y, z)$, for all $x, y, z \in P$. Now, since $d$ is surjective derivation, we see that $l(x, y, z) \subseteq d(l(x, y, z))$ for all $x, y, z \in P$.

Let $x, y, z \in P$ and $h \in l(x, y, z)$. Since $d$ is a surjective derivation, there exist $k \in P$ such that $d(k)=h$. By [12, Stat. 1 in Thm. 3.1] we get $h=d(k)=d(d(k)) \in d(l(x, y, z))$. Then $l(d(x), y, z) \subseteq d(l(x, y, z))$. Hence,

$$
l(u(l(d(x), y, z), l(x, d(y), z), l(x, y, d(z))) \subseteq d(l(x, y, z)) \quad \text { for all } \quad x, y, z \in P .
$$

This proves 2.1 is proved. In the same way we prove that

$$
l(d(u(x, y, z)))=l(u(d(x), d(y), d(z))) \quad \text { for all } \quad x, y, z \in P .
$$

The proof is complete.

As the following example shows, there exists a triple derivation on a poset which is not a surjective derivation.

Example 2.1. Let $(P, \leqslant)=(\{0,1,2\}, \leqslant)$. Define the function $d: P \rightarrow P$ by $d(0)=d(2)=0$ and $d(1)=1$. Clearl, $y d$ is a triple derivation on $P$. On the other hand, $d$ is not a derivation on $P$ since $d(l(1,2))=d(l(1))=\{0,1\}$ and $l(u(l(d(1), 2)), l(1, d(2)))=l(u(l(1,0)), l(1,0))=$ $l(u(l(0)))=l(0)=\{0\}$. Thus, $d$ is not a surjective derivation.

In what follows, $(P, \leqslant)$ is a poset.

Lemma 2.2. Let $d$ be a triple derivation on $P$, then the following statements hold:

1. $d(x) \leqslant x ;$ for all $x \in P$.

2. If $x \leqslant y$, then $d(x) \leqslant d(y)$;

3. If $I$ is an ideal of $P$, then $d(I) \subseteq I$;

4. If $P$ has the least element 0 then $d(0)=0$.

Proof. 1. Since $d$ is a triple derivation on $P$, we have

$$
d(l(x, x, x))=l(u(l(d(x), x, x), l(x, d(x), x)), l(x, x, d(x)))=l(u(l(d(x), x, x)))=l(d(x), x, x) .
$$

But

$$
d(l(x))=d(l(x, x, x))=l(d(x), x, x)=l(d(x), x) .
$$

On the other hand we have, $d(x) \in d(l(x))$, then $d(x) \in l(d(x), x)$. Hence $d(x) \leqslant x$.

2. Suppose that $x \leqslant y$, then

$$
l(d(u(x, y, y)))=l(d(u(y)))=l(u(d(x), d(y), d(y)))=l(u(d(x), d(y))) .
$$

But $d(x) \in l(u(d(x), d(y)))$, so $d(x) \in l(d(u(y)))$. Hence $d(x) \leqslant d(y)$.

3. Assume that $I$ is an ideal of $P$. If $x \in d(I)$, then there exist $t \in I$ such that $d(t)=x$. By Statement 1 , we get $d(t) \leqslant t$ so $x \leqslant t$, but $I$ is an ideal of $P$. Hence, $x \in I$. This proves that $d(I) \subseteq I$.

4. Let $P$ has the least element 0 then, by Statement 1 , we get $0 \leqslant d(0) \leqslant 0$. Hence, $d(0)=0$. The proof is complete.

Lemma 2.3. Let $d$ be a triple derivation on P. Then the following statements hold:

1. If $d(l(x))=l(y)$, then $d(x)=y$ 
2. If $d(u(x))=u(y)$, then $d(x)=y$.

Proof. 1. Let $d(l(x))=l(y)$, then by $y \in l(y)$ we get $y \in d(l(x))$. Then there exist $t \in l(x)$ such that $d(t)=y$. But $d(t) \leqslant d(x)$, so $y \leqslant d(x)$. On the other hand, $d(x) \in d(l(x))=l(y)$, so $d(x) \leqslant y$. Hence, $d(x)=y$.

2. Statement 2 can be proved in the same way as Statement 1.

Theorem 2.1. Let $P$ be a poset and $d: P \rightarrow P$ be a map. Then $d$ is a triple derivation on $P$ if and only if

1. $d(l(x, y, z))=l(d(x), y, z))=l(x, d(y), z)=l(x, y, d(z))$ for all $x, y, z \in P$;

2. $l(d(u(x, y, z)))=l(u(d(x), d(y), d(z)))$ for all $x, y, z \in P$.

Proof. We simply need to show that Condition 1 in Definition 2.1 is equivalent to Statement 1 in this theorem. First, assume that Statement 1 is satisfied, then

$$
d(l(x, y, z))=l(d(x), y, z)=l(u(l(d(x), y, z)))=l(u(l(d(x), y, z), l(x, d(y), z)), l(x, y, d(z))) .
$$

Assume, that $d$ is a triple derivation on $P$. Then

$$
l(d(x), y, z)=l(u(l(d(x), y, z))) \subseteq l(u(l(d(x), y, z), l(x, d(y), z)), l(x, y, d(z)))=d(l(x, y, z)) .
$$

On the other hand, suppose that $v \in d(l(x, y, z))$, then there exists $t \in l(x, y, z)$ satisfying $d(t)=v$. By Statements 1, 2 in Lemma 2.2, we have

$$
d(t) \leqslant d(x), \quad d(t) \leqslant d(y) \leqslant y, \quad d(t) \leqslant d(z) \leqslant z .
$$

This shows that $v=d(t) \in l(d(x), y, z)$. Hence, $d(l(x, y, z)) \subseteq l(d(x), y, z)$ and therefore, $d(l(x, y, z))=l(d(x), y, z)$.

In the same way we prove that

$$
d(l(x, y, z))=l(x, d(y), z), \quad d(l(x, y, z))=l(x, y, d(z)) .
$$

Lemma 2.4. Let $d$ be a triple derivation of $P$. Then $d(l(x, y, z))=l(d(x), d(y), d(z))$, for all $x, y, z \in P$.

Proof. First assume that $t \in l(d(x), d(y), d(z))$ and

$$
t \leqslant d(x), \quad t \leqslant d(y), \quad t \leqslant d(z) .
$$

By Statement 1 in Lemma $2.2, t \leqslant y$ and $t \leqslant z$. Then $t \in l(d(x), y, z)$. By Statement 1 in Theorem 2.1, $l(d(x), d(y), d(z)) \subseteq d(l(x, y, z))$. And vice versa, suppose that $t \in d(l(x, y, z))$, then there exists $v \in l(x, y, z)$ such that $d(v)=t$. Then by Statement 2 in Lemma 2.2 we get

$$
d(v) \leqslant d(x), \quad d(v) \leqslant d(y), \quad d(v) \leqslant d(z) .
$$

Hence, $t \in l(d(x), d(y), d(z))$ and $d(l(x, y, z)) \subseteq l(d(x), d(y), d(z))$. The proof is complete.

\section{FIXED POINTS OF A TRIPLE DERIVATIONS}

Throughout this section $P$ is a poset and $d$ is a triple derivation on $P$. We call $x$ a fixed point of $d$ in $P$ if $d(x)=x$. We also denote by $\operatorname{Fix}_{d}(P)=\{y \in P: d(y)=y\}$ the set of all fixed points of $d$ in $P$ and $d(P)=\{d(y): y \in P\}$.

Theorem 3.1. Let d be a triple derivation on $P$. Then

1. $d(x) \in \operatorname{Fix}_{d}(P)$, for all $x \in P$,

2. $\operatorname{Fix}_{d}(P)=d(P)$. 
Proof. 1. We are going to prove that $d(d(x))=d(x)$ for all $x \in P$. Let $x \in P$ then by Statement 1 in Lemma 2.2 and Statement 1 in Theorem 2.1 we have

$$
d(l(d(x)))=d(l(x, x, d(x)))=l(d(x), x, d(x))=l(d(x)) .
$$

Then by Statement 1 in Lemma 2.3 we obtain $d(d(x))=d(x)$. Hence, $d(x) \in \operatorname{Fix}_{d}(P)$.

2. By Statement $1, d(x) \in \operatorname{Fix}_{d}(P)$ for all $x \in P$, then $d(P) \subseteq \operatorname{Fix}_{d}(P)$. And vice versa, let $x \in \operatorname{Fix}_{d}(P)$, then $x=d(x)$. Hence, $\operatorname{Fix}_{d}(P) \subseteq d(P)$, and $\operatorname{Fix}_{d}(P)=d(P)$.

The proof is complete.

Lemma 3.1. Let $d, s$ be two triple derivations on $P$. Then $d=s$ if and only if $\operatorname{Fix}_{d}(P)=$ $\operatorname{Fix}_{s}(P)$.

Proof. It is obvious that as $d=s$, we have $\operatorname{Fix}_{d}(P)=\operatorname{Fix}_{s}(P)$. And vice versa, let $\operatorname{Fix}_{d}(P)=$ $\operatorname{Fix}_{s}(P)$, and $x \in P$. Since by Statement 1 in Theorem 3.1(1) we get $d(x) \in \operatorname{Fix}_{d}(P)=\operatorname{Fix}_{s}(P)$, then $s(d(x))=d(x)$. In the same way we confirm that $d(s(x))=s(x)$. Then by Statements 1,2 in Lemma 2.2 we get $d(x) \leqslant s(x)$ and $s(x) \leqslant d(x)$. Hence, $d(x)=s(x)$. The proof is complete.

Proposition 3.1. Let $d$ be a triple derivation on $P$ with the least element 0 . Then the following statement hold.

1. $0 \in \operatorname{Fix}_{d}(P)$,

2. If $x \in \operatorname{Fix}_{d}(P)$, and $y \leqslant x$, then $y \in \operatorname{Fix}_{d}(P)$,

3. If $P$ is directed, then, for each $x, y \in \operatorname{Fix}_{d}(P)$, there exists $z \in \operatorname{Fix}_{d}(P)$ satisfying $x \leqslant z$, $y \leqslant z$.

Proof. 1. Since $d(0)=0$, then $0 \in \operatorname{Fix}_{d}(P)$.

2. Assume that $x \in \operatorname{Fix}_{d}(P)$, and $y \leqslant x$ then $d(x)=x$. Then by Statement 1 in Theorem 2.1 and Statement 2 in Lemma 2.2 we get

$$
d(l(y))=d(l(x, y, y))=l(d(x), y, y)=l(x, y, y)=l(y) .
$$

Then by Statement 1 in Lemma 2.3(1) we get $d(y)=y$, and hence $y \in \operatorname{Fix}_{d}(P)$.

3. Suppose that $P$ is directed then for each $x, y \in P$ there exist $v \in P$ such that $x \leqslant v$ and $y \leqslant v$. Since $x, y \in \operatorname{Fix}_{d}(P)$, then $d(x)=x, d(y)=y$. But $d(x)=x \leqslant d(v)$ and $d(y)=y \leqslant d(v)$. We take $h=d(v)$ and by Statement 2 in Theorem 3.1 we get $z \in \operatorname{Fix}_{d}(P)$.

The proof is complete. $P$.

Corollary 3.1. If $P$ is a directed poset with the least element 0 , then $\operatorname{Fix}_{d}(P)$ is an ideal of

Theorem 3.2. Let $d$ be a triple derivation on $P$. Then, for all $x \in P$,

$$
\operatorname{Fix}_{d}(P) \cap l(x)=l(d(x)) .
$$

Proof. First assume that $y \in \operatorname{Fix}_{d}(P) \cap l(x)$, then $y=d(y)$ and $y \leqslant x$. Then $d(y) \leqslant d(x)$ and $y \leqslant d(x)$. Hence, $y \in l(d(x))$ an

$$
\operatorname{Fix}_{d}(P) \cap l(x) \subseteq l(d(x)) .
$$

On the other hand, we have $d(x) \leqslant x$ and $d(x) \in \operatorname{Fix}_{d}(P)$. Hence, $d(x) \in \operatorname{Fix}_{d}(P) \cap l(x)$. Then by Lemma 3.1 we get $l(d(x)) \subseteq \operatorname{Fix}_{d}(P) \cap l(x)$. Hence

$$
\operatorname{Fix}_{d}(P) \cap l(x)=l(d(x)) .
$$

The proof is complete. 


\section{IDEALS AND OPERATIONS RELATED WITH TRIPLE DERIVATIONS}

In this section, $P$ is a poset with the least element 0 .

Theorem 4.1. Let $d$ be a triple derivation on $P$. Then $\operatorname{Ker} d=\{x \in P: d(x)=0\}$ is a nonempty lower set of $P$.

Proof. By Statement 4 in Lemma 2.2 we have $d(0)=0$. Then $0 \in \operatorname{Ker} d$, and Ker $d \neq \phi$. Suppose that $x \in \operatorname{Ker} d$ and $y \leqslant x$ then $d(x)=0$. By Statement 2 in Lemma 2.2 we get $d(y) \leqslant d(x)=0$. Hence $d(y)=0$, and this shows that $y \in \operatorname{Ker} d$. The proof is complete.

Lemma 4.1. Let $d$ be a triple derivation on $P$ and $I$ be an ideal of $P$. Then $d^{-1}(I)$ is a nonempty lower set of $P$ such that $\operatorname{Ker} d \subseteq d^{-1}(I)$.

Proof. The identity $d(0)=0$ implies that $0 \in d^{-1}(I)$, and hence, $d^{-1}(I) \neq \phi$.

Assume that $x \in d^{-1}(I)$ and $y \leqslant x$, then $d(x) \in I$. Then by Statement 2 in Lemma 2.2 we get $d(y) \leqslant d(x) \in I$. Hence, $d(y) \in I$, and this shows that $y \in d^{-1}(I)$. Therefore, $d^{-1}(I)$ is a nonempty lower set of $P$. And vice versa, we observe that $\operatorname{Ker} d=d^{-1}(\{0\}) \subseteq d^{-1}(I)$. The proof is complete.

Lemma 4.2. Let $d$ be a triple derivation on $P$ and $I, J$ be two ideals of $P$. Then

1. if $I \subseteq J$, then $d(I) \subseteq d(J)$,

2. $d(I \cap J)=d(I) \cap d(J)$.

Proof. 1. Suppose that $x \in d(I)$, then there exist $y \in I \subseteq J$ such that $x=d(y)$. Then, $x \in d(J)$, and this shows that $d(I) \subseteq d(J)$.

2 It is clear that $d(I \cap J) \subseteq d(I) \cap d(J)$. And vice versa, let $x \in d(I) \cap d(J)$, then there exist $a \in I, b \in J$ such that $d(a)=x$ and $d(b)=x$. Then by Statement 1 in Theorem 2.1 we get

$$
d(l(a, a, d(b))=l(d(a), a, d(b))=l(x, a, x)=l(x) .
$$

But $x \in l(x)$ and hence, $x \in d(l(a, a, d(b)))$. Then there exist $z \in l(a, a, d(b))$ such that $d(z)=x$. By $z \leqslant a$ and $z \leqslant d(b) \leqslant b$ we see that $z \in I \cap J$. Hence $x \in d(I \cap J)$, and $d(I) \cap d(J) \subseteq d(I \cap J)$. The proof is complete.

Theorem 4.2. Let $d$ and $s$ be two triple derivations on $P$. Then $d$ and $s$ commute.

Proof. Let $d$ and $s$ BE two triple derivations on $P$. Then for each $x \in P$,

$$
d(l(s(x)))=d(l(s(x), x, x))=l(s(x), d(x), x)=l(s(x), d(x))
$$

and

$$
s(l(d(x)))=s(l(d(x), x, x))=l(d(x), s(x), x)=l(d(x)), s(x)) .
$$

Hence, $d(l(s(x)))=s(l(d(x)))$. But $d(s(x)) \in d(l(s(x)))$ and therefore, $d(s(x)) \in s(l(d(x)))$. Then there exists $z \in l(d(x))$ such that $d(s(x))=s(z)$. By Statement 2 in Lemma 2.2 we conclude that $s(z) \leqslant s(d(x))$, and therefore $d(s(x)) \leqslant s(d(x))$.

In the same way we can prove that $s(d(x)) \leqslant d(s(x))$. This yields the identity $d(s(x))=$ $s(d(x))$. The proof is complete.

Theorem 4.3. Let $d$ and $s$ be two triple derivations on $P$. Then $d \leqslant s$ if and only if $s d=d$.

Proof. Let $d$ and $s$ be two triple derivations on $P$ with $d \leqslant s$. Then, for any $x \in P$, we have $d(x) \in \operatorname{Fix}_{d}(P)$ i.e. $d(x)=d(d(x)) \leqslant s(d(x))$. Also by Statement 1 in Lemma 2.2 we have $s(d(x)) \leqslant d(x)$. Hence, $s(d(x))=d(x)$. This leads us to $s d=d$. On the other hand, thanks to Statement 2 in Lemma 2.2 , we have $d(x)=s(d(x)) \leqslant s(x)$ for each $x \in P$. This implies that $s d \leqslant s$. The proof is complete. 


\section{ACKNOWLEDGEMENTS.}

The author thanks the anonymous referee for critically reading the manuscript and furthermore for his valuable remarks, which significantly imroved the original version.

\section{REFERENCES}

1. M. Ashraf and A. Jabeen. Nonlinear generalized Lie triple higher derivation on triangular algebras // Bull. Iran. Math. Soc. 44:2, 513-530 (2018).

2. M. Ashraf, B.A. Wani and F. Wei. Multiplicative $*$-Lie triple higher derivations of standard operator algebras // Quaestiones Mathematicae. 42:7, 857-884 (2018).

3. T. Albu and M.L. Teply. Generalized deviation of posets and modular lattices // Discr. Math. 214:1-3, 1-19 (2000).

4. I. Chajda and J. Rachünek. Forbidden configurations for distributive and modular ordered sets // Order. 5:4, 407-423 (1989).

5. G. Gierz, K. H. Hofmann, K. Keimel, J.D. Lawson, M. Mislove and D.S. Scott. Continuous lattices and domains. Cambridge Univ. Press, Cambidge (2003).

6. M. Hongan and N.U. Rehman. A note on the generalized Jordan triple derivations on lee ideals in semiprime rings // Sarajevo J. Math. 9:21, 29-36 (2013).

7. W. Jing and S. Lu. Generalized Jordan derivations on prime rings and standared operator algebras // Taiwanese J. Math. 7:4, 605-613 (2003).

8. Y.S. Jung. Generalized Jordan higher triple derivations on prime rings // Indian J. Pure Appl. Math. 36:9, 513-524 (2005).

9. C.K. Liu and K.W. Shiue. Generalized Jordan triple $(\theta, \phi)$-derivations on semiprime rings // Taiwanese J. Math. 11:5, 1397-1406 (2007).

10. Z. Ullah, I. Javaid and M.A. Chaudhry. On generalized triple derivations on lattices // ARS Combinatoria. 113, 463-471 (2014).

11. X.L. Xin, T.Y. Li and J.H. Lu. On derivations of lattices // Information Sci. 178:2, 307-316 (2008).

12. H. Zhang and Q. Li. On derivations of partially ordered sets // Math. Slovaca. 67:1, 17-22 (2017).

Ahmed Yunis Abdelwanis,

Department of Mathematics,

Faculty of Science,

Cairo University,

Giza,12613 Egypt

E-mail: ayunis@sci.cu.edu.eg 